\title{
The functional anatomy of semantic retrieval is influenced by gender, menstrual cycle, and sex hormones
}

\author{
C. Konrad $\cdot$ A. Engelien $\cdot$ S. Schöning $\cdot$ P. Zwitserlood $\cdot$ A. Jansen · \\ E. Pletziger $\cdot$ P. Beizai $\cdot$ A. Kersting $\cdot$ P. Ohrmann $\cdot$ E. Luders $\cdot$ \\ R. R. Greb · W. Heindel · V. Arolt $\cdot$ H. Kugel
}

Received: 2 March 2008/ Accepted: 17 May 2008/Published online: 12 June 2008

(C) The Author(s) 2008

\begin{abstract}
This study examines the neurobiology of semantic retrieval and describes the influence of gender, menstrual cycle, and sex hormones on semantic networks. Healthy right-handed subjects (12 men, 12 women) were investigated with 3T-fMRI during synonym generation. Behavioral performance and sex hormone levels were assessed. Women were examined during the early follicular and midluteal cycle phase. The activation pattern in all groups involved left frontal and temporal as well as bilateral medial frontal, cingulate, occipital, basal ganglia, and cerebellar regions. Men showed greater left frontal activation than women in both menstrual cycle phases. Women yielded high correlations of left prefrontal activation with estradiol in the midluteal phase and with progesterone in both phases. Testosterone levels correlated highly with left prefrontal activation in all three groups. In all, we describe a cerebral network involved in semantic processing and
\end{abstract}

Konrad and Engelien contributed equally to the present work.

C. Konrad $(\bowtie) \cdot$ A. Engelien · S. Schöning · E. Pletziger ·

P. Beizai - A. Kersting · P. Ohrmann · V. Arolt

Department of Psychiatry and Psychotherapy,

University of Muenster, Albert-Schweitzer-Str.11,

48149 Muenster, Germany

e-mail: c.konrad@uni-muenster.de

C. Konrad · A. Engelien - S. Schöning $\cdot$ E. Pletziger $\cdot$ P. Beizai Interdisciplinary Center for Clinical Research (IZKF),

University of Muenster, Muenster, Germany

P. Zwitserlood

Department of Clinical Psychology, University of Muenster,

Muenster, Germany

A. Jansen

Department of Psychiatry, University Hospital Aachen,

Aachen, Germany demonstrate that it is significantly affected by gender and sex steroid hormones.

Keywords Cognition .

Functional magnetic resonance imaging .

Sex steroid hormones - Language - Gender characteristics . Gender differences

\section{Introduction}

Semantic retrieval is a memory process involving word meaning. It entails several cognitive processes such as perceiving a target word, holding it online in working memory, retrieving its meaning, activating related concepts and, depending on the task at hand, actively searching for concepts with equivalent meaning. To date, semantic retrieval has primarily been investigated in the context of word-fluency or category-fluency tasks, in which subjects

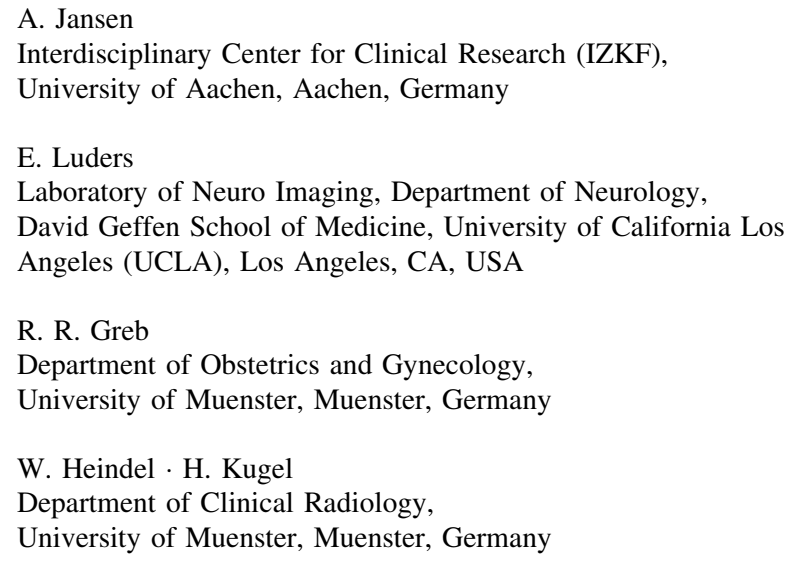


have to produce a list of members of a category. Functional imaging investigations on semantic fluency tasks emphasize the role of the left inferior frontal and premotor cortex in a network including cingulate gyrus, thalamus, basal ganglia, and cerebellum (Audenaert et al. 2000; Basho et al. 2007; Gourovitch et al. 2000; Hirshorn and Thompson-Schill 2006; Klein et al. 1995; Nyberg et al. 2002).

Semantic processing has often been related to gender or hormonal influences, however the results are heterogeneous. Behavioral studies yielded variable results on the influence of gender in semantic tasks. Some authors reported higher verbal performance in women (Kimura and Clarke 2002; Weiss et al. 2006; Wirth et al. 2007) while others could not detect differences (Roberts and Bell 2002). Likewise, the influence of menstrual cycle (Gordon and Lee 1993; Rode et al. 1995) and sex steroid hormones is controversially discussed (Carlson and Sherwin 1998; Roberts and Bell 2002; Thilers et al. 2006; Yonker et al. 2003). Using functional imaging, some investigators observed physiological correlates of gender differences in semantic processing, e.g. higher activation in women than men (Baxter et al. 2003; Jaeger et al. 1998; Ragland et al. 2000) or vice versa (Bell et al. 2006; Buckner et al. 1995; Gizewski et al. 2006; Rossell et al. 2002) (for details see "Discussion"). Others were unable to confirm these findings (Gur et al. 2000; Halari et al. 2005, 2006; Schlosser et al. 1998; Sommer et al. 2004; Weiss et al. 2003). Some of these controversies on gender differences may relate to effects of sex steroid hormones and menstrual cycle, which are not taken into consideration in most imaging studies. While one functional imaging study failed to find hormonal influences on a rhyming decision with word pairs (Veltman et al. 2000), Fernandez et al. demonstrated hormone-, task-, and region-specific variations across the menstrual cycle in women performing a semantic decision task. Women in the midluteal phase showed higher activation compared to women in the perimenstrual phase (Fernandez et al. 2003). This is in agreement with Dietrich et al. who described a more global influence of cycle phase on magnitude and extent of activations during word-stem-completion with the most pronounced signal increases in the midluteal phase (Dietrich et al. 2001). Influences of gender, menstrual cycle, and sex steroid hormones are not restricted to semantic functions, though. Our group recently reported sex differences in a spatial working-memory task that were related to testosterone and estrogen levels. Men showed stronger activation than women in the left inferior parietal lobe, left fusiform gyrus and lingual gyrus. Testosterone correlated with brain activation in men and women in the low estrogen phase, while estrogen showed large correlations with brain activation in both women groups, indicating that sex hormones might indeed contribute to fMRI variability in cognitive tasks (Schöning et al. 2007).
In the present study, we apply a synonym generation task requiring silent retrieval of as many synonyms as possible while striving for high synonym quality, emphasizing the semantic challenge of the task. To the best of our knowledge, this is the first study taking into account the influences of gender, menstrual cycle phases, and sex hormone levels on activation patterns in fMRI during semantic retrieval. The main objective of this study is to characterize cerebral networks during synonym generation. In addition, we hypothesize that brain activation patterns are influenced by gender, phases of the female menstrual cycle, and serum levels of estrogen, progesterone, and testosterone. Based on previous findings, we conjecture that women outperform men and show higher levels of brain activation. We also expect higher activations in the midluteal than in the perimenstrual phase. To explore if sex steroid hormones exert an independent effect, hormone levels will be correlated with brain activation within each group separately.

\section{Material and methods}

\section{Subjects}

Thirty-four healthy, right-handed, heterosexual German subjects (age-range 23-45 years) participated in the study after giving their informed consent. The study was approved by the local Institutional Ethical Review Board and was performed in accordance with the ethical standards laid down in the 1964 Declaration of Helsinki. Men were examined once, women twice in pseudo-randomized order-once 1-3 days after onset of menses (early follicular phase) and once in the midluteal phase adjusted for cycle length (individual cycle length -7 days with a range of \pm 2 days). Only premenopausal women with stable cycle length were included and any form of hormonal treatment was excluded. The phase of the menstrual cycle was confirmed by sex steroid hormone analysis (for details see below). None of the subjects had a history of a serious medical disease, or any neurological or psychiatric illness. Twelve females and 12 males were included in the final analysis (for details see Table 1).

Reasons of exclusion were either technical or to achieve homogeneity of the hormonal status and cycle phase. A second fMRI scan could not be obtained for two women. In six female participants, the sex steroid hormone analysis revealed values not corresponding to the target phase of their menstrual cycle that was expected from their menstrual history (four anovulatory cycles, two cycles with high progesterone levels in the presumed early follicular phase). Two males were excluded because of unusually low or high testosterone levels.

Intelligence was assessed using CFT 20 (Weiss 1998) and MWT-B (Lehrl et al. 1995), two German standard 
Table 1 Subjects baseline characterization and task performance (mean \pm SD)

\begin{tabular}{|c|c|c|c|}
\hline & $\begin{array}{l}\text { Women during early } \\
\text { follicular phase }\end{array}$ & $\begin{array}{l}\text { Women during } \\
\text { midluteal phase }\end{array}$ & Men \\
\hline Number of subjects & 12 & 12 & 12 \\
\hline Age & $30.8 \pm 5.0$ & $30.8 \pm 5.0$ & $33.2 \pm 6.2$ \\
\hline Intelligence, more visuo-spatially based estimate measure (CFT) & $118.3 \pm 13.7$ & $118.3 \pm 13.7$ & $124.0 \pm 10.2$ \\
\hline Intelligence, more verbally based estimate measure (MWT-B) & $112.0 \pm 13.6$ & $112.0 \pm 13.6$ & $117.0 \pm 11.9$ \\
\hline Testosterone in $\mathrm{ng} / \mathrm{ml}^{\mathrm{a}}$ & $0.34 \pm 0.13$ & $0.37 \pm 0.13$ & $5.10 \pm 1.45^{*}$ \\
\hline Estradiol in $\mathrm{pg} / \mathrm{ml}^{\mathrm{b}}$ & $46.75 \pm 11.10^{*}$ & $129.00 \pm 56.81^{*}$ & $36.0 \pm 6.30^{*}$ \\
\hline Progesterone in $\mathrm{ng} / \mathrm{ml}^{\mathrm{c}}$ & $0.65 \pm 0.15$ & $10.02 \pm 5.70^{*}$ & $0.70 \pm 0.20$ \\
\hline Reproduction Quantity score & $47.7 \pm 21.0$ & $48.3 \pm 18.9$ & $50.8 \pm 22.3$ \\
\hline Reproduction Quality score & $9.3 \pm 4.0$ & $9.8 \pm 3.1$ & $10.2 \pm 2.8$ \\
\hline Reproduction Quality/quality ratio & $0.23 \pm 0.12$ & $0.24 \pm 0.14$ & $0.23 \pm 0.09$ \\
\hline Novel generation Quantity score & $40.6 \pm 19.3$ & $44.8 \pm 16.8$ & $44.7 \pm 15.7$ \\
\hline Novel generation Quality score & $4.7 \pm 1.4$ & $6.6 \pm 2.1$ & $5.9 \pm 2.0$ \\
\hline Novel generation Quality/quality ratio & $0.16 \pm 0.15$ & $0.17 \pm 0.09$ & $0.14 \pm 0.06$ \\
\hline
\end{tabular}

$T$ tests between groups are not significant if not indicated otherwise. ${ }^{*}$ Value differs significantly from value in other groups

a For women during the early follicular phase and women during the midluteal phase versus men $P<0.001$

${ }^{\mathrm{b}}$ For women during the early follicular phase versus men $P=0.008$. For women during the midluteal phase versus men $P<0.001$ and versus women during the early follicular phase $P<0.001$

${ }^{\mathrm{c}}$ For women during the midluteal phase versus men $P<0.001$ and versus women during the early follicular phase $P<0.001$

short measures for estimating intelligence quotients based on visuo-spatial and verbal material, respectively. There were no significant differences between men and women for age or intelligence measures (see Table 1). All participants had more than 12 years of education. Handedness was assessed by the Edinburgh handedness inventory (Oldfield 1971).

Sex steroid hormone assessment

Before fMRI scanning, a venous blood sample was drawn to determine total testosterone, estradiol, progesterone, luteinizing (LH) and follicle-stimulating hormone (FSH). This was always done between 2 and $5 \mathrm{pm}$ in order to minimize known day-time variation (Chappell 2005; Davison and Bell 2006). Men were excluded from further analysis if serum testosterone levels were below $2.3 \mathrm{ng} / \mathrm{ml}$ and above $8 \mathrm{ng} / \mathrm{ml}$. Women were diagnosed as being in the early follicular phase of menstrual cycle if onset of menstrual bleeding had started and if serum estradiol and progesterone levels were $\leq 70 \mathrm{pg} / \mathrm{ml}$ and $<1 \mathrm{ng} / \mathrm{ml}$, respectively. They were categorized as being in the midluteal phase of menstrual cycle if onset of menstrual bleeding had occurred at individual cycle length minus 7 days ( \pm 2 days) prior to the examination. All included women had estradiol levels $\geq 66 \mathrm{pg} / \mathrm{ml}$ and progesterone levels $\geq 4 \mathrm{ng} / \mathrm{ml}$. FSH and LH levels were within the normal range of premenopausal women in all female subjects $(\mathrm{FSH}<15 \mathrm{IU} / \mathrm{L}, \mathrm{LH}<$ $20 \mathrm{IU} / \mathrm{L})$. All hormone analyses were performed by electrochemiluminescence immunoassays (ECLIA, Roche Diagnostics, Mannheim, Germany, as described in http:// wwwlabor.uni-muenster.de/easy/easynavfa.htm).

\section{Experimental design}

During fMRI scanning, five synonym generation condition blocks $\left(S_{1-5}\right)$ were administered, each consisting of $30 \mathrm{~s}$ task performance, followed by a $30 \mathrm{~s}$ rest period $(R)$, according to an $S_{1}-R-S_{2}-R-S_{3}-R-S_{4}-R-S_{5}-R$ design. Synonym generation is considered a task that reflects both semantic retrieval and verbal fluency. During each block, two German adjectives were presented using Presentation Software ${ }^{\circledR}$ (Version 0.81, 2004, Neurobehavioral Systems Inc., Albany, CA, USA) on an MR compatible back-projection screen. Word frequency of adjectives was balanced between blocks (Celex Lexical Database for German, Release 2, Version 2.5, cf. (Baayen et al. 1995)). Subjects were instructed to silently retrieve as many synonyms as possible until the appearance of a new word or until a fixation cross indicated the beginning of the rest period. The instruction further urged subjects to strive for quality of the synonyms. These data were obtained as part of a larger protocol. Women were tested twice, receiving two equivalent versions of the synonym retrieval task in a counter-balanced order in their early follicular and midluteal phase of the menstrual cycle. Each of the versions was randomly administered to $50 \%$ of the men. 
Synonym retrieval was performed again after fMRI scanning, with subjects speaking aloud to obtain behavioral measures. Adjectives were presented on a computer screen and responses were recorded. This post-scanning synonym task was performed on the ten previously seen adjectives, aiming to probe performance in the scanner (reproduction condition). Subjects were also tested on seven new adjectives to test the de novo generation of synonyms (novel generation condition).

\section{Analysis of behavioral data}

Two types of analyses were performed on the behavioral responses to assess quantity and quality of the retrieved synonyms. Total word count served as global quantity score. A synonym quality score was obtained by categorizing responses according to their status as (near) synonyms. This was done by checking their occurrence in two synonym dictionaries (Müller 1997; Wahrig 2002). Quality-to-quantity ratios indicate the proportion of synonyms relative to the total of produced words. Thus, the following performance measures were established: a Quantity score, a Quality score, a Qualityquantity-ratio both for the reproduction and the novel generation conditions (see Table 1). Differences between men and the women in both phases were assessed using unpaired $t$ tests, while differences between women as a function of menstrual cycle were tested using paired $t$ test.

\section{MRI data acquisition}

MRI data were acquired in a $3 \mathrm{~T}$ whole body scanner (Intera T30, Philips, Best, NL) equipped with master gradients (nominal gradient strength $30 \mathrm{mT} / \mathrm{m}$, maximal slew rate $150 \mathrm{mT} / \mathrm{m}$ per millisecond). A circularly polarized transmit/receive birdcage head coil with a HF reflecting screen at the cranial end was used for spin excitation and resonance signal acquisition. Functional images were acquired using a $\mathrm{T}_{2}^{*}$ weighted single shot echo-planar (EPI) sequence (whole brain coverage, $\mathrm{TE}=50 \mathrm{~ms}, \mathrm{TR}=3,000$ $\mathrm{ms}$, flip angle $90^{\circ}$, slice thickness $3.6 \mathrm{~mm}$ without gap, matrix $64 \times 64$, FOV $230 \mathrm{~mm}$, in-plane resolution $3.6 \times$ $3.6 \mathrm{~mm}$ ). Thirty-six transversal slices orientated parallel to the $\mathrm{AC}-\mathrm{PC}$ line were taken.

\section{MRI data analysis}

Functional MRI data were analyzed using SPM2 standard routines and templates (http://www.fil.ion.ucl.ac.uk/spm). To allow for saturation effects of the BOLD signal, the first ten images of each session ( $30 \mathrm{~s}$ pre-stimulus interval) were discarded. The remaining images were realigned, normalized and resliced to a voxel size of $2 \times 2 \times 2 \mathrm{~mm}^{3}$, and smoothed with a $9 \mathrm{~mm}$ kernel. Data were then filtered with a high-pass filter (cut-off period of $128 \mathrm{~s}$ ). BOLDresponses for synonym generation were modeled using a boxcar function convolved with the canonical hemodynamic response function employed by SPM2. To obtain activation maps across subjects, the functional data were combined in a random-effects analysis to identify brain areas activated during synonym generation. In a first level fixed-effects analysis, we obtained one statistical parametric map (SPM) and corresponding contrast images for each subject, reflecting the contrasts of interest. These individual contrast images were entered into a second-level random-effects analysis to determine activations at a group level. The second-level analysis consisted of three steps:

Step 1: Within-group activation patterns were assessed by one sample $t$ tests in a random-effects analysis. The alpha error was set to $P<0.05$ corrected (false discovery rate) and the contiguity threshold to $\geq 20$ voxels. To allow comparability with other studies, in particular to assure independence from sample sizes, $T$ values were converted into Cohen's $d$ effect sizes according to Cohen's $d=2 \tilde{t} / \sqrt{(d f)}$ (Cohen 1998; Kline 2004), using the vbm2 toolbox provided by C. Gaser, University of Jena, Germany (http://dbm.neuro.uni-jena.de). $T$ and $d$ values were overlaid on a single-subject template provided in MRIcron (Rorden et al. 2007). Anatomical localizations of activated brain regions were identified using the MSU toolbox based on the Talairach daemon (Lancaster et al. 2000).

Step 2: Differences between groups were calculated within the regions that showed activation in the group results (step 1), using an ANOVA model. The alpha error was set to $P<0.001$ uncorrected and the contiguity threshold to $\geq 20$ voxels. To assure comparability across studies, differences are reported as $T$ and Cohen's $d$ values and were overlaid on a single-subject template provided in MRIcron (see above).

Step 3: The correlation of brain activation with hormone levels for estradiol, progesterone, and testosterone was investigated in areas activated in the group results (from step 1). Simple regression (correlation) analysis was performed for testosterone, estrogen, and progesterone. For descriptive analysis, unthresholded $T$ values were converted into correlation coefficients $r$ using the cg_t2x.m script provided by C. Gaser, University of Jena, Germany (http://dbm.neuro.uni-jena.de). The corresponding $P$ values were determined from $r$ values using the online calculator for scientists at Graphpad Software (http://www.graphpad.com/quickcalcs/ pvalue1.cfm, degrees of freedom $=10$ ). Correlation coefficients (and equivalent $P$ values) were overlaid on a 
single-subject template provided in MRIcron (Rorden et al. 2007).

\section{Results}

Task performance

There were no significant differences between males and females in both phases of the menstrual cycle in any of the post hoc performance measures. Small differences between means, such as higher scores for men during word reproduction, or higher scores for midluteal women during novel word generation, were within the range of the SD. During the reproduction condition, subjects produced an average word quantity of $48.9 \pm 20.2$ words, of which only $9.8 \pm$ 3.3 words met the criteria for synonym quality, corresponding to a Quality-quantity-ratio of $0.23 \pm 0.12$. During the novel generation condition, the average number of words generated to novel target adjectives was $43.3 \pm$ 16.9 with $5.7 \pm 2.0$ synonyms, corresponding to a Qualityquantity-ratio of $0.16 \pm 0.10$ (for details see Table 1).

\section{Within-group activation patterns during synonym generation}

The synonym retrieval task led to prominently left lateralized prefrontal activation. Both men and women showed extensive activations on the lateral surface of the frontal cortex, comprising the left inferior and middle frontal gyri, the precentral gyrus, and the right inferior frontal gyrus. On the medial surface of the hemispheres, bilateral activation of superior and medial frontal gyrus (supplementary motor area, SMA) and the anterior cingulate gyrus were noted. Another consistent pattern of activation was observed in the middle occipital gyrus and lingual gyrus, as expected due to the baseline visual and reading components of the task. In addition, parts of the basal ganglia and cerebellum were active bilaterally (for details see Fig. 1; Table 2).

Fig. 1 Brain activation during synonym generation in women during the early follicular phase of the menstrual cycle, women during the midluteal phase of the menstrual cycle, and men. Statistical $T$ maps and $d$ maps for effect sizes are rendered on a single-subject template in MNI space (thresholded at an alphaerror level of $P<0.05$ corrected (false discovery rate), contiguity threshold of $\geq 20$ voxels)
Between-group activation patterns during synonym generation

Group comparisons demonstrated significantly more activation of the left middle frontal and left precentral gyrus in men than in women in either phase (Figs. 1, 2; Table 3). The difference between men and women in their early follicular phase was larger (cluster size 155 voxels) than the difference between men and women in their midluteal phase (cluster size 63 voxels). Women showed a trend towards higher activation of prefrontal regions when in their midluteal phase, which did not reach statistical significance (Fig. 1).

Correlation of brain activation with sex steroid hormone measurements

For the correlation between brain areas activated by the task with sex hormone levels, correlation coefficients in each voxel are displayed in Fig. 3.

Estradiol showed high correlations with left prefrontal activations for women in their midluteal phase $(r=0.6, P=$ 0.039). Estradiol correlated only weakly with left prefrontal activation when women were in the early follicular phase $(r<0.3, P=0.343)$ and in men $(r<0.2, P=0.533)$.

Progesterone showed high correlations with left prefrontal activation in women both in the early follicular $(r=$ $0.6, P=0.039)$ and the midluteal phase $(r=0.7, P=0.011)$, while correlations in men were weak $(r<0.2, P=0.533)$.

Testosterone values were strongly correlated with left prefrontal activation in all three groups, that is men $(r=$ $0.6, P=0.039)$, women in their early follicular phase $(r=$ $0.5, P=0.098)$, and women in their midluteal phase $(r=$ $0.7, P=0.011)$.

\section{Discussion}

The main objective of this study was the characterization of cerebral networks during synonym generation. We also
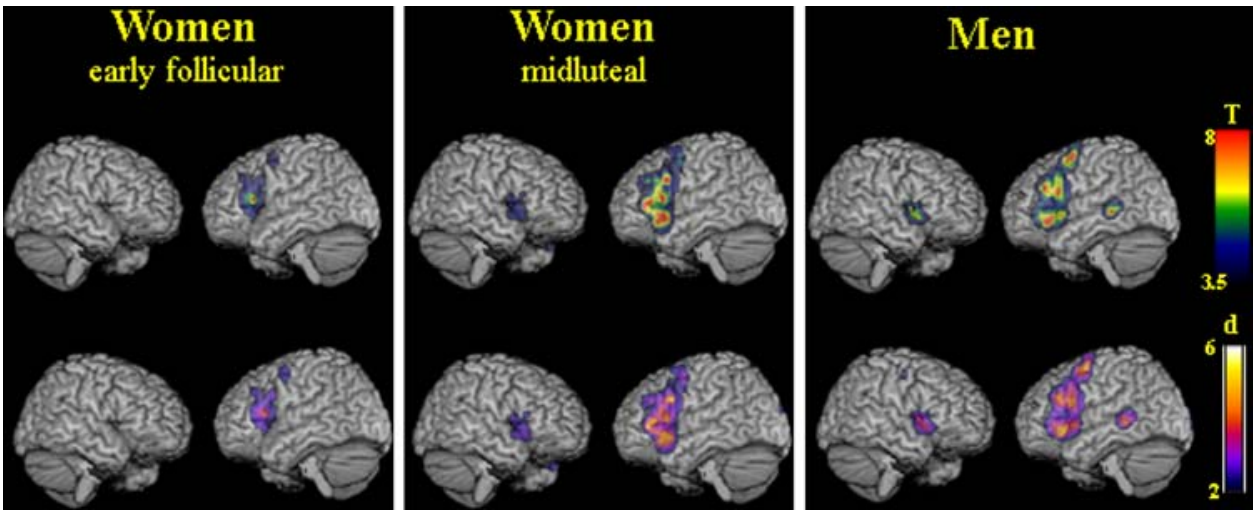


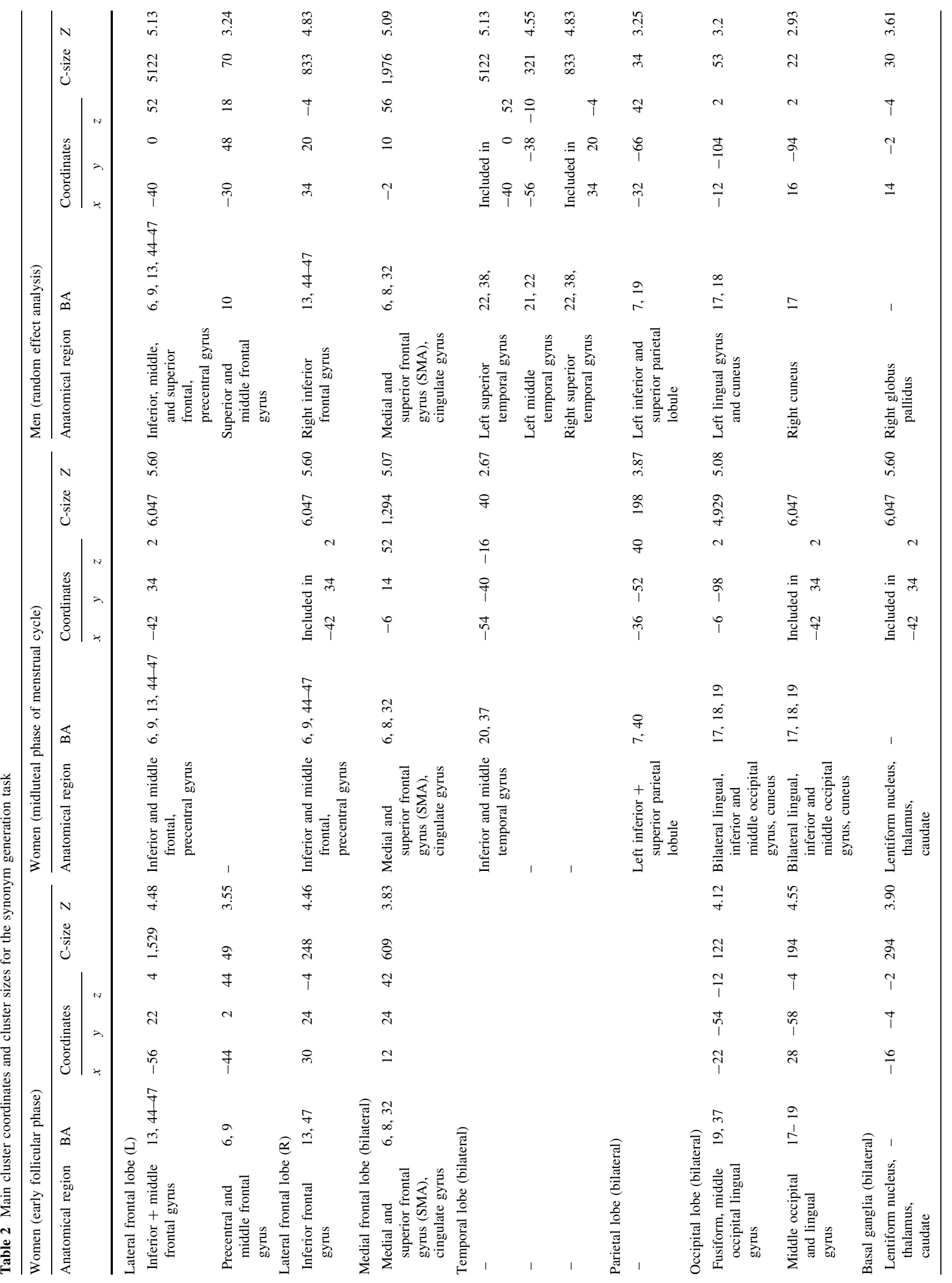



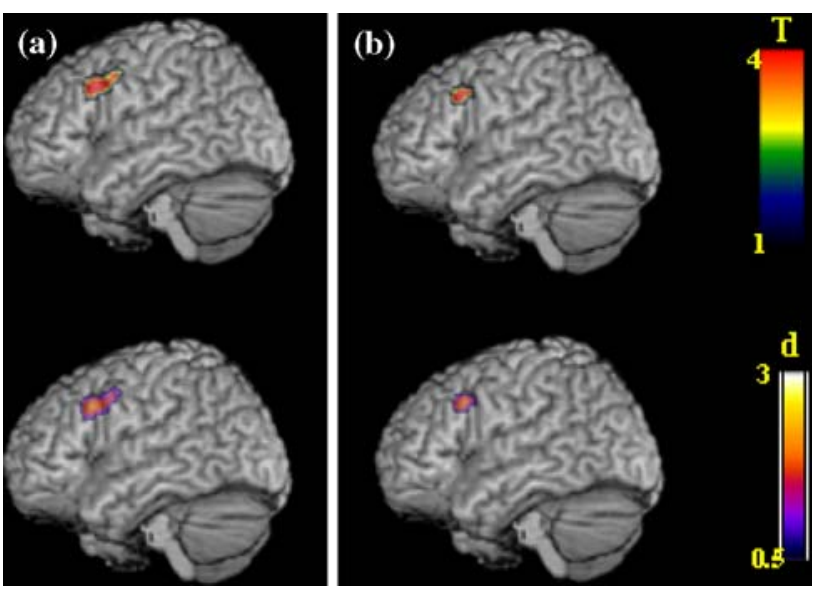

Fig. 2 Differences in the left dorsolateral prefrontal cortex activation in (a) men compared to women during the early follicular phase of the menstrual cycle, and (b) men compared to women during the midluteal phase of the menstrual cycle. Statistical $T$ maps and $d$ maps for effect sizes are rendered on a single-subject template in MNI space (thresholded at an alpha-error level of $P<0.001$ uncorrected, contiguity threshold of $\geq 20$ voxels)

hypothesized that brain activation patterns are influenced by gender, phases of the female menstrual cycle, and serum levels of sex steroid hormones. We observed activation of the classical language and memory areas. In the absence of behavioral differences, men showed higher brain activation in left frontal language areas than women. Women showed a trend towards higher activation in the midluteal phase. The correlation of brain activation with sex hormone levels was different between groups and across the menstrual cycle.

Within-group activation patterns during synonym generation

Synonym generation has rarely been investigated using functional imaging techniques, while word generation, word and category fluency tasks have been investigated more often. Left lateralized activation of the frontal cortex, including precentral, inferior, and middle frontal gyri is characteristic for word generation (Klein et al. 1995; Nyberg et al. 2002). Left frontal areas are also active when words are semantically associated (Buckner et al. 1995; Chee et al. 1999; Chou et al. 2006a, b; Gabrieli et al. 1998; Price 2000; Woodward et al. 2006), or when words are pronounced silently (Bookheimer 2002; Borowsky et al. 2005; Huang et al. 2002). SMA and cingulate gyrus are involved in word generation and semantic retrieval (Etard et al. 2000; Papathanassiou et al. 2000). The anterior inferior temporal gyrus has been associated with lexical retrieval (Brunswick et al. 1999), and the left posterior middle temporal lobe with semantic processing, such as the association of objects on the basis of their meaning (Chou 
Table 3 Significantly different activations between groups during synonym generation as obtained by a random-effects analysis $(P<0.001$ uncorrected, cluster size $\geq 20$ voxels)

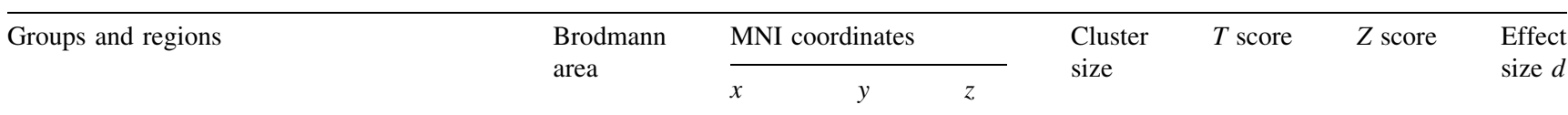

Men versus women in the early follicular phase

\begin{tabular}{llllllll} 
(a) Left middle frontal and precentral gyrus & 9 & -40 & 18 & 32 & 155 & 4.67 & 3.85 \\
& & -40 & 4 & 40 & & 3.94 & 2.82 \\
$\begin{array}{l}\text { Men versus women in the midluteal phase } \\
\text { (b) Left middle frontal and precentral gyrus }\end{array}$ & 9 & -40 & 20 & 32 & 63 & 4.50 & 3.75 \\
\hline
\end{tabular}

Men showed higher activation in left middle and precentral gyrus than (a) women in the early follicular phase and (b) women in the midluteal phase

Fig. 3 Correlation of sex hormone levels with brain activation during synonym generation in women during the early follicular phase of the menstrual cycle, women during the midluteal phase of the menstrual cycle, and men. Maps for correlation coefficients $r$ are rendered on a single-subject template in MNI ( $r$ from 0.2 to 1 corresponds to $P$ from 0.533 to $<0.001)$

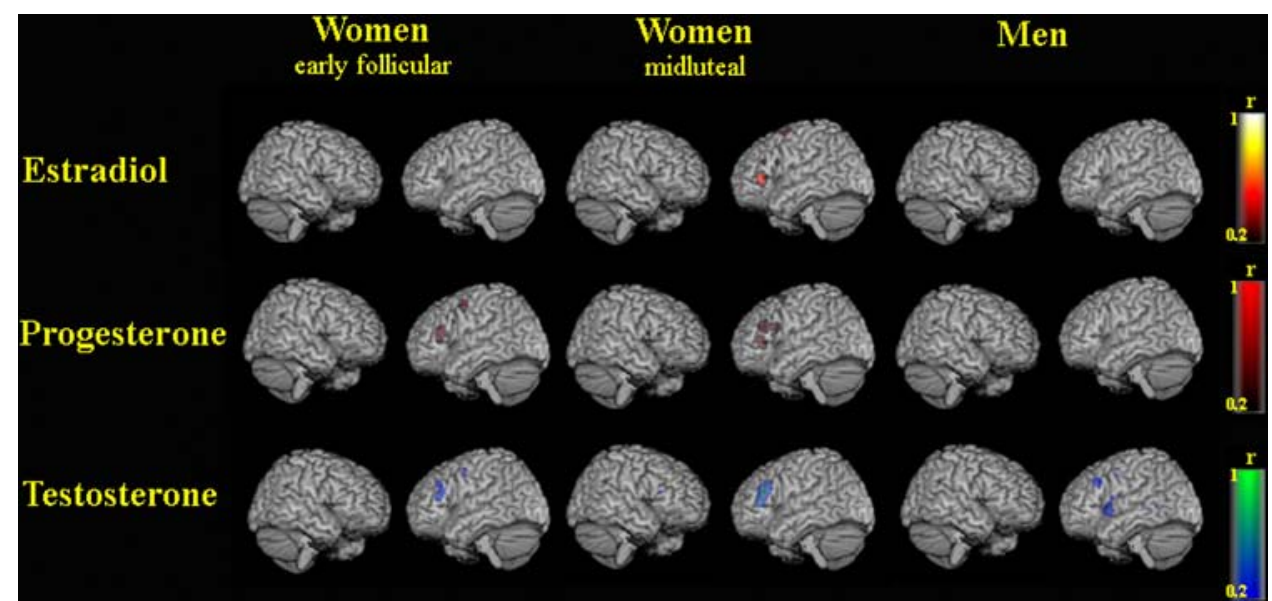

et al. 2006a; Price 2000; Woodward et al. 2006). Cerebellar activation has previously been reported in silent speech generation and synonym generation as well (Jansen et al. 2005; Klein et al. 1995; Nyberg et al. 2002). The synonym generation paradigm, thus far only used sparsely in combination with functional imaging techniques, provides stable patterns of brain activation with predominant activation of left prefrontal language regions.

\section{Gender effects during synonym generation}

In accordance with our hypothesis, we observed genderrelated differences in the degree of brain activation in the key left frontal language areas. The difference in activation between men and women was most pronounced in the left middle frontal and precentral gyri. It was most pronounced when men were compared to women during the early follicular phase of their cycle. The gender differences reported here cannot be attributed to differences in verbal performance in our fMRI subject sample, as behavioral performance did not significantly differ between groups on any measure. Buckner and colleagues reported a PET study in which brain activation during verb generation was larger in magnitude in men than in women. While hormonal status of women was not reported, this result is in accordance with our present study (Buckner et al. 1995). Gizewski et al. replicated these results comparing men and women, more specifically, women in their midluteal phase (Gizewski et al. 2006). In a smaller sample, Halari et al. observed no significant differences in brain activation between men and women using a letter fluency task (Halari et al. 2006). In accordance with a meta-analysis including 377 men and 442 women (Sommer et al. 2004), synonym generation was strongly left-lateralized in men and women.

\section{Menstrual cycle effects during synonym generation}

Only few studies have so far attempted to measure the influence of fluctuating sexual hormone levels across the menstrual cycle on fMRI activation patterns. Some behavioral observations point towards an effect of sexual hormones on cognitive functions (Hampson 1990; Hausmann and Gunturkun 2000), but this is discussed controversially (Gordon and Lee 1993; Halari et al. 2005; Roberts and Bell 2002). Our current results, while obtained from a rather small sample size, do not support the hypothesis of performance differences in the measures applied. Our imaging results might indicate that menstrual 
cycle effects on fMRI activation patterns are unrelated to task performance, thus favoring a more biological explanation. This would be in accordance with an earlier and smaller study of Dietrich et al. who investigated six male and six female subjects during a word-stem-completion task, a mental rotation task, and a motor task (Dietrich et al. 2001). In both cognitive tasks, the most pronounced increases of BOLD signal were observed in women during the peak estrogen condition (midluteal phase), while no significant differences occurred between male and female subjects in the low estrogen condition. This is in line with the trend observed in the present study. Fernandez et al. investigated 12 women, but no men, with a synonymjudgment task. In agreement with our data, they also observed higher activation during the midluteal phase (Fernandez et al. 2003). In a study on emotional word processing, medio-orbitofrontal activity was also increased in the midluteal phase (Protopopescu et al. 2005). In contrast, Veltman et al. investigated eight women and could not find influences of estrogen on a rhyming decision on word pairs. This lack of effect could possibly be attributed to the choice of the activation paradigm (phonological rather than semantic), and the small sample size (Veltman et al. 2000). While we observed a trend towards higher left prefrontal activation, this trend did not reach statistic significance. However, this trend is in line with the data obtained by Dietrich et al. (2001), Fernandez et al. (2003) and Protopopescu et al. (2005). The lack of significance could imply that menstrual cycle does not exert any effects on BOLD signal, but given the data by others, it is more plausible that the effects are too small to reach statistic significance with group sizes of 12 subjects. We thus conclude that fMRI studies on semantic tasks should pay attention to menstrual cycle effects if group sizes exceed 12 subjects per group.

\section{Correlation of brain activation with sex steroid} hormone measurements

A major strength of this study is that sex hormone concentrations were assessed right before scanning. We were thus able to correlate sex hormone levels with brain activation during task performance. Interestingly, correlations depended on gender and phase of the menstrual cycle: estradiol correlations with brain activation were high in the high estrogen phase of the menstrual cycle, while progesterone correlations were significant in both phases investigated here. Testosterone correlated strongly with brain activation in all three groups. Our results underscore the potential influence of sex hormones on neurocognitive processes. The role of sex steroid hormones is extensively discussed for memory-related tasks, first and foremost in the context of hormone substitution in aging or demented populations. Effects for both testosterone (Driscoll and Resnick 2007; Geerlings et al. 2006; Zitzmann 2006) and estradiol (Craig and Murphy 2007; Doren 2007; Genazzani et al. 2007) have been reported for different cognitive domains. While our results are in line with findings on spatial working memory (Schöning et al. 2007), findings on verbal memory are more ambiguous, reporting diverse effects of estrogen and/or testosterone (Carlson and Sherwin 2000; Cherrier et al. 2005; Martin et al. 2007; Moffat et al. 2000; Thilers et al. 2006). In particular, the influence of sex hormones on behavioral performance is controversial. While some authors report behavioral effects (Hampson 1990; Hausmann and Gunturkun 2000) others do not (Gordon et al. 1986; Gordon and Lee 1993; Halari et al. 2005; Mumenthaler et al. 2001; Roberts and Bell 2002). Our results also do not reveal performance differences in the behavioral measures applied here. Nevertheless, the results of the present study suggest that sex steroid hormones exert more direct effects on fMRI BOLD-signal changes which are unrelated to task performance. Our results fit well with the above-mentioned earlier studies on other verbal tasks, with the most pronounced increases of BOLD signal in women during the peak estrogen (midluteal) phase (Dietrich et al. 2001; Fernandez et al. 2003; Protopopescu et al. 2005).

Physiological mechanisms

Obviously, fMRI does not allow to directly investigate the biological and molecular action mechanisms underlying different BOLD responses in men and women, as a function of the phases of the menstrual cycle, and of sex hormone levels. Thus, sex hormone action on cerebral neurons or vascular effects would both be consistent with our results. Sex steroid hormones might exert direct effects on cerebral neurons, reflected by altered brain activation by many different routes. These effects might be mediated by intracellular neural estrogen receptors such as ER-alpha and ER-beta, but also by dendritic, presynaptic, or glial estrogen receptors, by ligand-gated ion channels, by effects on gene expression or on neural excitability, by secondmessenger systems, or by interactions with the neurotransmitter systems. Alternatively or in addition, the neurovascular coupling might be altered by estrogeninduced cerebral vasodilatation and blood-flow changes. Estrogen might increase the concentration of vasodilatory nitric oxide (NO) through an Akt/PKB-enzyme dependent pathway and the endothelial isoform of nitric oxide synthase (eNOS). Although our data cannot clarify the mechanisms of action, they reveal an influence of gender, phase of menstrual cycle, and sex hormone levels on brain activation. Our results suggest that differences in brain activation do not solely depend on estrogen levels, as we 
found a clear-cut difference between men and women in their low estrogen phase. This can only be explained by a combined effect of sex steroid hormones. Indeed, correlations with progesterone in the female groups, and particularly testosterone in all three groups, emphasize the role of all three hormones on fMRI activation during synonym retrieval.

\section{Limitations}

Some limitations of our study should be mentioned. Rigorous exclusion of subjects with abnormal sex hormone levels restricted the sample size to 12 subjects per group, limiting statistical power. Further, behavioral measures had to be acquired outside the scanner to avoid artifacts related to motor or verbal responses during functional image acquisition. As in many imaging study, cognitive effort required for task performance could not be measured. Nevertheless, we aimed at obtaining objective performance measures for synonym retrieval reflecting within-scanner performance by examining both repeated and novel target items. In particular, the cautious control of hormonal levels is unique to the present fMRI study design.

This study demonstrates a cerebral network involved in semantic retrieval that is influenced by gender and sex steroid hormones.

Acknowledgments This work was supported by the Interdisciplinary Center for Clinical Research (IZKF), University of Muenster, Germany (FG 4). The 2004 DGPPN/AstraZeneca Förderpreis to A.E. is also gratefully acknowledged.

Open Access This article is distributed under the terms of the Creative Commons Attribution Noncommercial License which permits any noncommercial use, distribution, and reproduction in any medium, provided the original author(s) and source are credited.

\section{References}

Audenaert K, Brans B, Van Laere K et al (2000) Verbal fluency as a prefrontal activation probe: a validation study using 99mTc-ECD brain SPET. Eur J Nucl Med 27:1800-1808

Baayen RH, Piepenbrock R, Gulikers L (1995) The CELEX lexical database for German (CD-ROM). Linguistic data consortium. University of Pennsylvania, Philadelphia

Basho S, Palmer ED, Rubio MA et al (2007) Effects of generation mode in fMRI adaptations of semantic fluency: paced production and overt speech. Neuropsychologia 45:1697-1706

Baxter LC, Saykin AJ, Flashman LA et al (2003) Sex differences in semantic language processing: a functional MRI study. Brain Lang 84:264-272

Bell EC, Willson MC, Wilman AH et al (2006) Males and females differ in brain activation during cognitive tasks. Neuroimage 30:529-538

Bookheimer S (2002) Functional MRI of language: new approaches to understanding the cortical organization of semantic processing. Annu Rev Neurosci 25:151-188
Borowsky R, Owen WJ, Wile TL et al (2005) Neuroimaging of language processes: fMRI of silent and overt lexical processing and the promise of multiple process imaging in single brain studies. Can Assoc Radiol J 56:204-213

Brunswick N, McCrory E, Price CJ et al (1999) Explicit and implicit processing of words and pseudowords by adult developmental dyslexics: a search for Wernicke's Wortschatz? Brain 122(Pt 10): 1901-1917

Buckner RL, Raichle ME, Petersen SE (1995) Dissociation of human prefrontal cortical areas across different speech production tasks and gender groups. J Neurophysiol 74:2163-2173

Carlson LE, Sherwin BB (1998) Steroid hormones, memory and mood in a healthy elderly population. Psychoneuroendocrinology 23:583-603

Carlson LE, Sherwin BB (2000) Higher levels of plasma estradiol and testosterone in healthy elderly men compared with age-matched women may protect aspects of explicit memory. Menopause $7: 168-177$

Chappell PE (2005) Clocks and the black box: circadian influences on gonadotropin-releasing hormone secretion. J Neuroendocrinol 17:119-130

Chee MW, O'Craven KM, Bergida R et al (1999) Auditory and visual word processing studied with fMRI. Hum Brain Mapp 7:15-28

Cherrier MM, Matsumoto AM, Amory JK et al (2005) The role of aromatization in testosterone supplementation: effects on cognition in older men. Neurology 64:290-296

Chou TL, Booth JR, Bitan T et al (2006a) Developmental and skill effects on the neural correlates of semantic processing to visually presented words. Hum Brain Mapp 27(11):915-924

Chou TL, Booth JR, Burman DD et al (2006b) Developmental changes in the neural correlates of semantic processing. Neuroimage 29:1141-1149

Cohen J (1998) Statistical power analysis for the behavioral sciences, 2 edn. Erlbaum, Hillsdale

Craig MC, Murphy DG (2007) Oestrogen, cognition and the maturing female brain. J Neuroendocrinol 19:1-6

Davison SL, Bell R (2006) Androgen physiology. Semin Reprod Med 24:71-77

Dietrich T, Krings T, Neulen J et al (2001) Effects of blood estrogen level on cortical activation patterns during cognitive activation as measured by functional MRI. Neuroimage 13:425-432

Doren M (2007) Cognitive function and menopausal hormone therapy. Hum Reprod Update 13:329; author reply 329-330

Driscoll I, Resnick SM (2007) Testosterone and cognition in normal aging and Alzheimer's disease: an update. Curr Alzheimer Res 4:33-45

Etard O, Mellet E, Papathanassiou D et al (2000) Picture naming without Broca's and Wernicke's area. NeuroReport 11:617-622

Fernandez G, Weis S, Stoffel-Wagner B et al (2003) Menstrual cycledependent neural plasticity in the adult human brain is hormone, task, and region specific. J Neurosci 23:3790-3795

Gabrieli JD, Poldrack RA, Desmond JE (1998) The role of left prefrontal cortex in language and memory. Proc Natl Acad Sci USA 95:906-913

Geerlings MI, Strozyk D, Masaki K et al (2006) Endogenous sex hormones, cognitive decline, and future dementia in old men. Ann Neurol 60:346-355

Genazzani AR, Pluchino N, Luisi S et al (2007) Estrogen, cognition and female ageing. Hum Reprod Update 13:175-187

Gizewski ER, Krause E, Wanke I et al (2006) Gender-specific cerebral activation during cognitive tasks using functional MRI: comparison of women in mid-luteal phase and men. Neuroradiology 48:14-20

Gordon HW, Lee PA (1993) No difference in cognitive performance between phases of the menstrual cycle. Psychoneuroendocrinology 18:521-531 
Gordon HW, Corbin ED, Lee PA (1986) Changes in specialized cognitive function following changes in hormone levels. Cortex 22:399-415

Gourovitch ML, Kirkby BS, Goldberg TE et al (2000) A comparison of rCBF patterns during letter and semantic fluency. Neuropsychology 14:353-360

Gur RC, Alsop D, Glahn D et al (2000) An fMRI study of sex differences in regional activation to a verbal and a spatial task. Brain Lang 74:157-170

Halari R, Hines M, Kumari V et al (2005) Sex differences and individual differences in cognitive performance and their relationship to endogenous gonadal hormones and gonadotropins. Behav Neurosci 119:104-117

Halari R, Sharma T, Hines M et al (2006) Comparable fMRI activity with differential behavioural performance on mental rotation and overt verbal fluency tasks in healthy men and women. Exp Brain Res 169:1-14

Hampson E (1990) Variations in sex-related cognitive abilities across the menstrual cycle. Brain Cogn 14:26-43

Hausmann M, Gunturkun O (2000) Steroid fluctuations modify functional cerebral asymmetries: the hypothesis of progesteronemediated interhemispheric decoupling. Neuropsychologia 38:1362-1374

Hirshorn EA, Thompson-Schill SL (2006) Role of the left inferior frontal gyrus in covert word retrieval: neural correlates of switching during verbal fluency. Neuropsychologia 44:25472557

Huang J, Carr TH, Cao Y (2002) Comparing cortical activations for silent and overt speech using event-related fMRI. Hum Brain Mapp 15:39-53

Jaeger JJ, Lockwood AH, Van Valin RD Jr et al (1998) Sex differences in brain regions activated by grammatical and reading tasks. Neuroreport 9:2803-2807

Jansen A, Floel A, Van Randenborgh J et al (2005) Crossed cerebrocerebellar language dominance. Hum Brain Mapp 24:165-172

Kimura D, Clarke PG (2002) Women's advantage on verbal memory is not restricted to concrete words. Psychol Rep 91:1137-1142

Klein D, Milner B, Zatorre RJ et al (1995) The neural substrates underlying word generation: a bilingual functional-imaging study. Proc Natl Acad Sci USA 92:2899-2903

Kline RB (2004) Beyond significance testing: reforming data analysis methods in behavioral research. American Psychological Association, Washington DC

Lancaster JL, Woldorff MG, Parsons LM et al (2000) Automated Talairach atlas labels for functional brain mapping. Hum Brain Mapp 10:120-131

Lehrl S, Triebig G, Fischer B (1995) Multiple choice vocabulary test MWT as a valid and short test to estimate premorbid intelligence. Acta Neurol Scand 91:335-345

Martin DM, Wittert G, Burns NR et al (2007) Testosterone and cognitive function in ageing men: data from the Florey Adelaide Male Ageing Study (FAMAS). Maturitas 57:182-194

Moffat SD, Zonderman AB, Harman SM et al (2000) The relationship between longitudinal declines in dehydroepiandrosterone sulfate concentrations and cognitive performance in older men. Arch Intern Med 160:2193-2198

Müller W (1997) Der Duden, Bd. 8. Synonymwörterbuch der deutschen Sprache. Duden Verlag, Mannheim

Mumenthaler MS, O'Hara R, Taylor JL et al (2001) Relationship between variations in estradiol and progesterone levels across the menstrual cycle and human performance. Psychopharmacology (Berl) 155:198-203

Nyberg L, Forkstam C, Petersson KM et al (2002) Brain imaging of human memory systems: between-systems similarities and within-system differences. Brain Res Cogn Brain Res 13:281292
Oldfield RC (1971) The assessment and analysis of handedness: the Edinburgh inventory. Neuropsychologia 9:97-113

Papathanassiou D, Etard O, Mellet E et al (2000) A common language network for comprehension and production: a contribution to the definition of language epicenters with PET. Neuroimage 11:347357

Price CJ (2000) The anatomy of language: contributions from functional neuroimaging. J Anat 197(Pt 3):335-359

Protopopescu X, Pan H, Altemus M et al (2005) Orbitofrontal cortex activity related to emotional processing changes across the menstrual cycle. Proc Natl Acad Sci USA 102:16060-16065

Ragland JD, Coleman AR, Gur RC et al (2000) Sex differences in brain-behavior relationships between verbal episodic memory and resting regional cerebral blood flow. Neuropsychologia 38:451-461

Roberts JE, Bell MA (2002) The effects of age and sex on mental rotation performance, verbal performance, and brain electrical activity. Dev Psychobiol 40:391-407

Rode C, Wagner M, Gunturkun O (1995) Menstrual cycle affects functional cerebral asymmetries. Neuropsychologia 33:855-865

Rorden C, Karnath HO, Bonilha L (2007) Improving lesion-symptom mapping. J Cogn Neurosci 19:1081-1088

Rossell SL, Bullmore ET, Williams SC et al (2002) Sex differences in functional brain activation during a lexical visual field task. Brain Lang 80:97-105

Schlosser R, Hutchinson M, Joseffer S et al (1998) Functional magnetic resonance imaging of human brain activity in a verbal fluency task. J Neurol Neurosurg Psychiatry 64:492-498

Schöning S, Engelien A, Kugel $\mathrm{H}$ et al (2007) Influences of sex, menstrual cycle, and sex steroids hormones on cortical activation patterns during mental rotation in BOLD-based fMRI. Neuropsychologia 45(14):3203-3214

Sommer IE, Aleman A, Bouma A et al (2004) Do women really have more bilateral language representation than men? A metaanalysis of functional imaging studies. Brain 127:1845-1852

Thilers PP, Macdonald SW, Herlitz A (2006) The association between endogenous free testosterone and cognitive performance: a population-based study in 35 to 90 year-old men and women. Psychoneuroendocrinology 31:565-576

Veltman DJ, Friston KJ, Sanders G et al (2000) Regionally specific sensitivity differences in fMRI and PET: where do they come from? Neuroimage 11:575-588

Wahrig G (2002) Deutsches Wörterbuch. Bertelsmann Lexikon Verlag, Gütersloh

Weiss EM, Siedentopf C, Hofer A et al (2003) Brain activation pattern during a verbal fluency test in healthy male and female volunteers: a functional magnetic resonance imaging study. Neurosci Lett 352:191-194

Weiss EM, Ragland JD, Brensinger CM et al (2006) Sex differences in clustering and switching in verbal fluency tasks. J Int Neuropsychol Soc 12:502-509

Weiss RH (1998) CFT 20. Grundintelligenztest Skala 2 (CFT 20) mit Wortschatztest (WS) und Zahlenfolgentest (ZF), 4 edn. Hogrefe, Goettingen

Wirth M, Horn H, Koenig T et al (2007) Sex differences in semantic processing: event-related brain potentials distinguish between lower and higher order semantic analysis during word reading. Cereb Cortex 17:1987-1997

Woodward TS, Meier B, Cairo TA et al (2006) Temporo-prefrontal coordination increases when semantic associations are strongly encoded. Neuropsychologia 44:2308-2314

Yonker JE, Eriksson E, Nilsson LG et al (2003) Sex differences in episodic memory: minimal influence of estradiol. Brain Cogn $52: 231-238$

Zitzmann M (2006) Testosterone and the brain. Aging Male 9:195199 